\title{
A Rare Case of Infectious Costochondritis Following Breast Surgery: Pseudomonal Infection Related Costochondritis
}

\author{
Seok Won Hong ${ }^{1}$, Da Woon Lee ${ }^{1}$, Hwan Jun Choi ${ }^{1,2}$ \\ ${ }^{1}$ Department of Plastic and Reconstructive Surgery and ${ }^{2}$ Institute of Tissue Regeneration, Soonchunhyang University College of Medicine, Cheonan, Korea
}

\begin{abstract}
Breast augmentation is among the most commonly performed cosmetic surgical procedures. Infections that arise after breast implant surgery can range from simple wound infections to periprosthetic infections. The published management algorithms for infection following breast augmentation indicate a need for surgeon awareness of complications, including infection, seroma, skin necrosis, device exposure, and capsular contracture. This paper reports a rare case of infective costochondritis following breast surgery in a 31-year-old woman that progressed to a continuous inflammatory course after implant removal.
\end{abstract}

Keywords: Surgical wound infection; Mammaplasty; Breast; Tietze's syndrome

\section{Introduction}

Breast augmentation is one of the most commonly performed cosmetic procedures [1]. The most popularly accepted material used in breast augmentation is the silicone implant [2]. As with any surgery, implant-based breast augmentation is associated with a number of risks and complications. Although uncommon, costochondritis and Tietze syndrome are among these potential complications. van Schalkwyk and van Wingerden [3], reported a rare variant case of Tietze syndrome, which presented with severe chest pain localized to the costosternal region several months after breast surgery [4]. Infectious costochondritis has also been reported as a rare complication after breast surgery [5]. These two complications can be easily confused. Unless infectious signs are definite, a patient complaining of costochondral pain due to infectious costochondritis after recent breast surgery could easily be misdiagnosed as having this variant of Tietze syndrome. Although infective costochondritis requires surgical procedures, Tietze syndrome can be managed by conservative treatment such as nonsteroidal anti-inflammatory drugs [6].

As with infective costochondritis, inflammatory costochondritis cases have also been reported in patients after breast reconstruction [3,5]. However, to the best of our knowledge there have been no reports of infective costochondritis after augmentation mammoplasty. This is a rare case report of a pseudomonal infection of the costochondral junction after breast implant removal. The patient provided written informed consent for the publication and the use of her images.

\section{Case}

A healthy 31-year-old woman with no significant medical history or operative history
Received: January 21, 2019

Revised: February 20, 2019

Accepted: February 20, 2019

\section{Corresponding author:}

Hwan Jun Choi, M.D., Ph.D.

Department of Plastic and Reconstructive Surgery, Soonchunhyang University Cheonan Hospital, Soonchunhyang University College of Medicine, 31 Suncheonhyang 6-gil, Dongnamgu, Cheonan 31151, Korea

Tel: +82-41-570-2195

Fax: +82-41-574-6133

E-mail: medi619@hanmail.net

This work was supported by the Soonchunhyang University Research Fund.

This is an Open Access article distributed under the terms of the Creative Commons Attribution Non-Commercial License (http://creativecommons.org/licenses/by-nc/4.0/) which permits unrestricted non-commercial use, distribution, and reproduction in any medium, provided the original work is properly cited.

C) 2019 Korean Wound Management Society 
of the anterior chest wall underwent augmentation mammoplasty with silicone implants via the inframammary fold approach at a local clinic. Round shape, textured type $200 \mathrm{~mL}$ implants were inserted into the submuscular plane of both breasts. Not long after the operation, she felt pain and discomfort at the surgical site. Although infection was not certain, the patient strongly wanted the implants removed due to severe pain, and these were immediately removed at the same local clinic. With post-implant removal prophylactic antibiotics and dressing changes, her symptoms were relieved without any other specific complications and the patient returned to normal life. One month after the removal of implants, she was referred to Soonchunhyang University Cheonan Hospital with turbid discharge from the surgical site. Chest discomfort with heat sensation of the inframammary fold was accompanied with marginal induration and tenderness (Fig. 1). She was immediately admitted to the department of plastic surgery, followed by overall laboratory examination and magnetic resonance imaging (MRI). Her C-reactive protein levels were elevated at $95 \mathrm{mg} / \mathrm{L}$ (normal range, $0-5.0 \mathrm{mg} / \mathrm{L}$ ) and mild leukocytosis $\left(11.1 \times 10^{9} / \mathrm{L}\right)$ was shown. MRI revealed an enhancing lesion appearing through the skin, pectoralis major muscle, and chest wall on the lower portion of the right breast (Fig. 2). Surgical debridement was performed under general anesthesia. Intraoperatively, turbid discharge was observed and irrigated; on gross examination, granulation tissue was noted in the pectoralis major and minor muscles and perichondrium of the costal cartilage (Fig. 3). The costal cartilage, especially in the costochondral junction, was discolored by a yellowish dis-

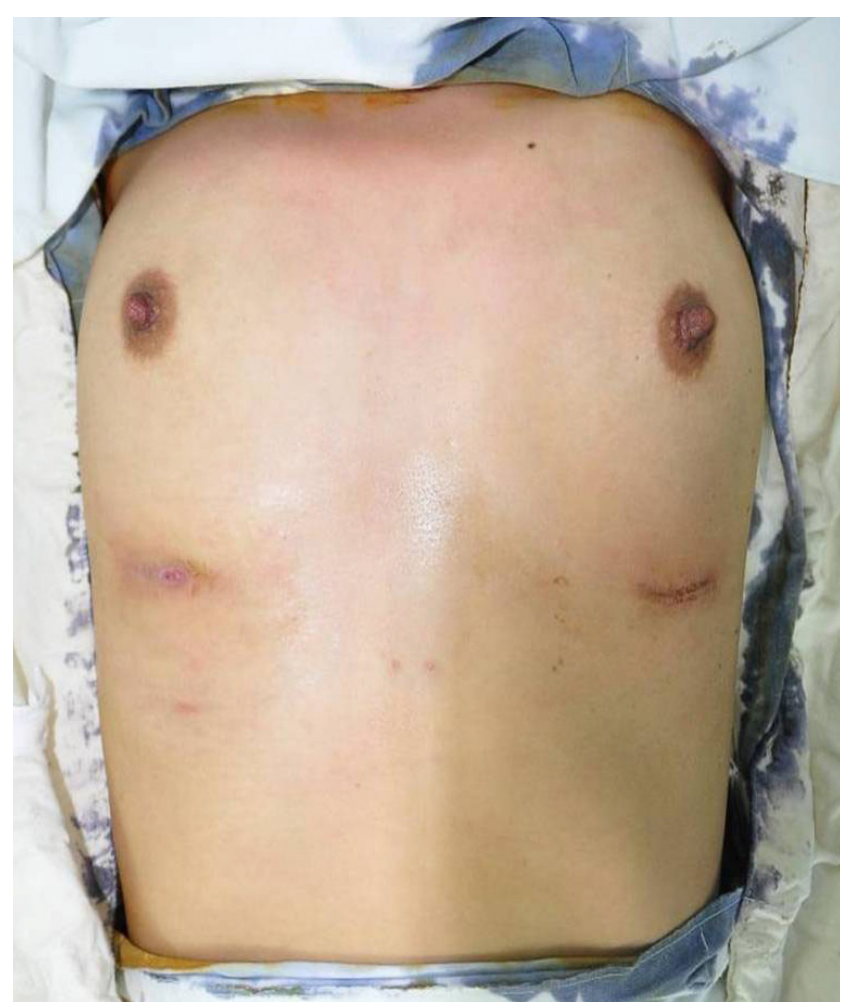

Fig. 1. Preoperative photograph of the surgical site. Heat sensation of the inframammary fold was accompanied with marginal induration and tenderness.
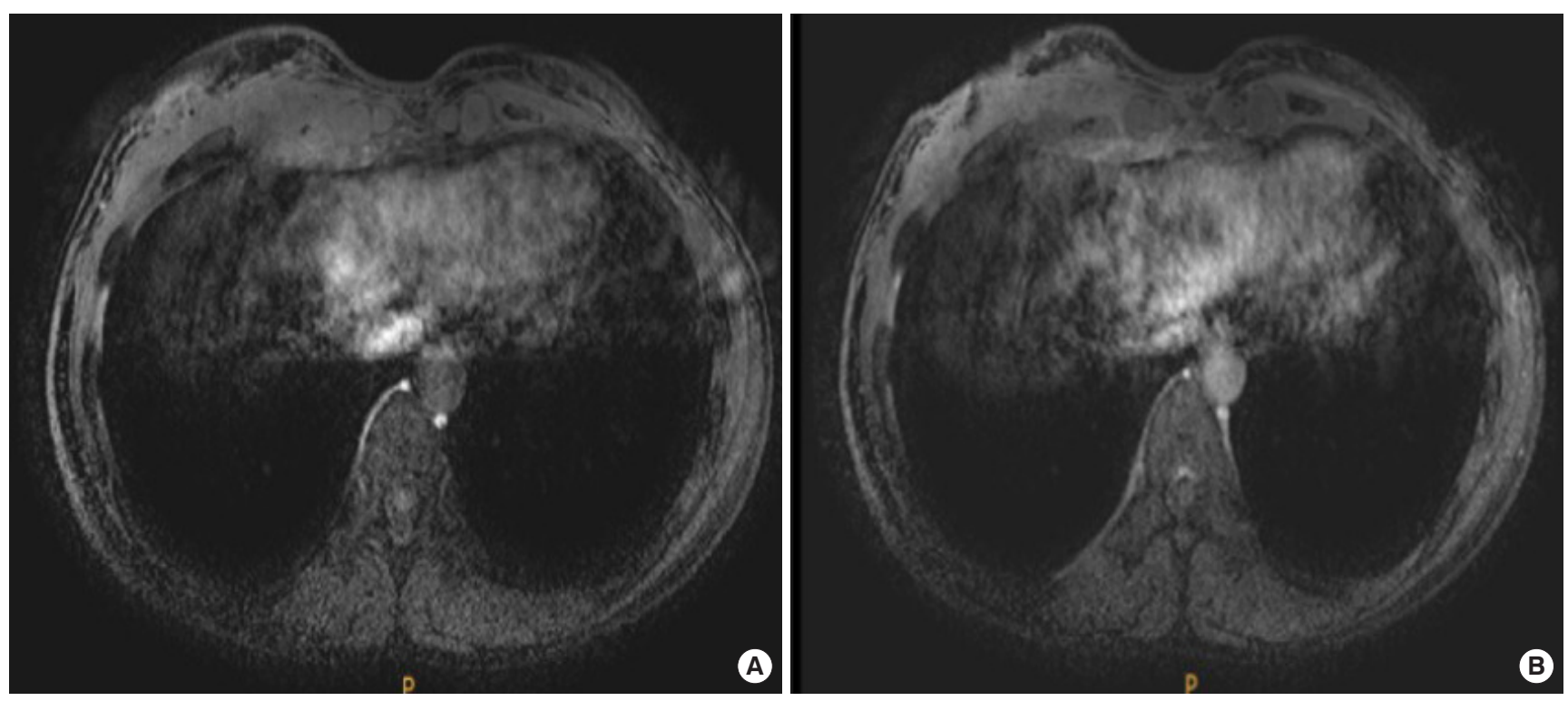

Fig. 2. Preoperative magnetic resonance image (MRI) images. (A) Enhancing lesions through the skin, pectoralis major muscle, and chest wall on the lower portion of the right breast. (B) Enhancing lesions involving the costal cartilage. 
charge. The partial-thickness infected portion of the costal cartilage was focally carved out and removed. Histologic examination of the removed tissue showed that the cartilage was damaged by suppurative inflammation (Fig. 4). Tissue culture results showed that the costal cartilage was infected by Pseu-

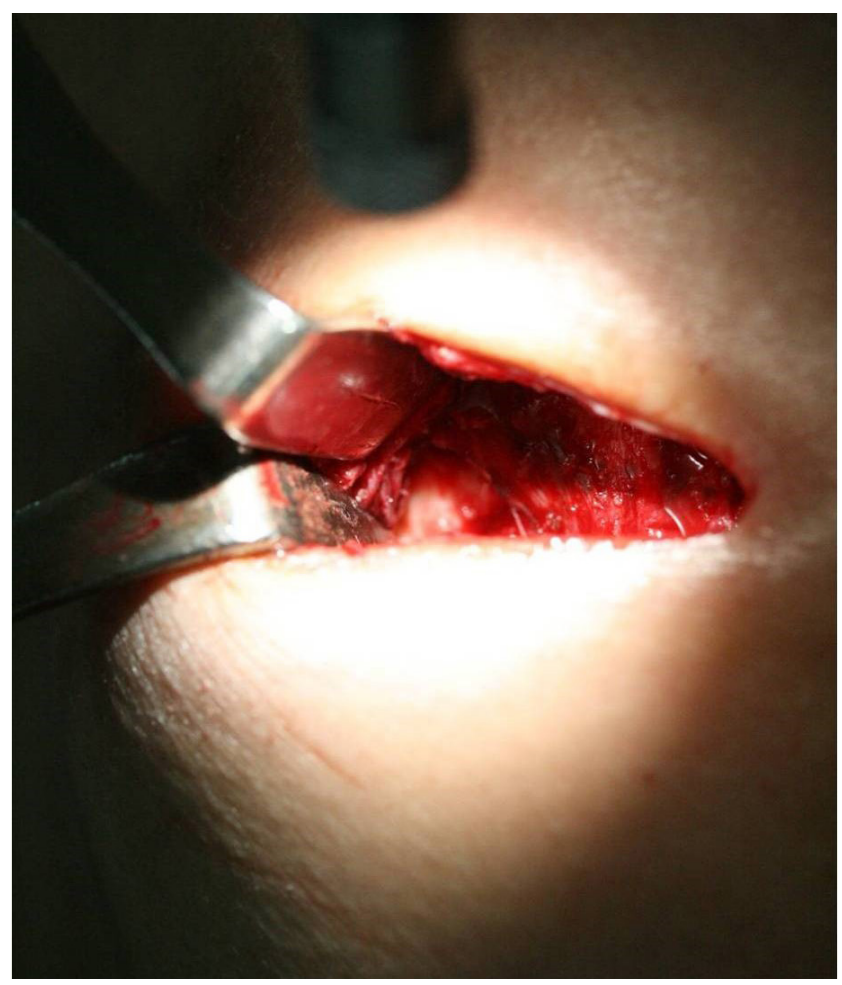

Fig. 3. Intraoperative photograph of infective costochondritis. Images showing a clear perichondrium on the left side and dirty granulation tissue with fibrosis on the right side involving the pectoralis muscle and costal cartilage.

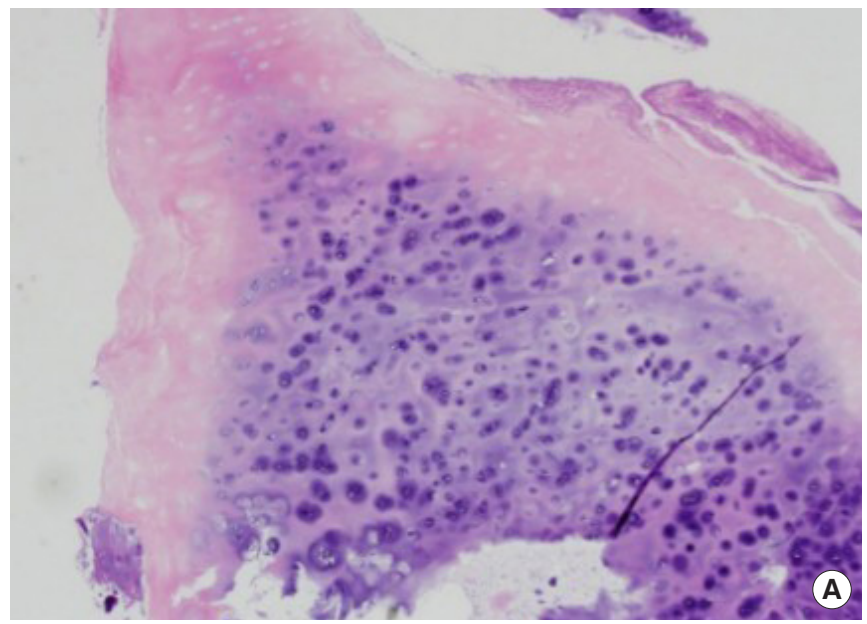

domonas aeruginosa. In close consultation with the department of infectious disease, intravenous carbapenem was administered $500 \mathrm{mg}$ twice a day for 2 weeks. Suction drain was removed 2 days after the operations, and the patient was treated successfully with postoperative wound management and antibiotics. She was discharged 2 weeks after the operation, and her postoperative course at 6 months was uncomplicated with no evidence of recurrence or complications such as wound dehiscence or a palpable scar.

\section{Discussion}

Costochondritis is a common self-limiting condition defined as inflammation of the costochondral junctions or chondrosternal joints, seen in patients presenting to the outpatient clinic and emergency department $[7,8]$. Diagnosis relies on patient history and physical examination. In general, costochondritis occurs on multiple levels and lacks swelling or induration [8]. Palpation of the affected cartilage segments produces pain which radiates on the chest wall [9]. Clinicians should exclude the possibility of coronary or gastrointestinal disease and carefully take the patient's traumatic, medical and surgical history for diagnosis [8].

Once costochondritis has been diagnosed, it is important to differentiate between inflammatory and infective causes, because the treatment for each is completely different. The former is self-limiting and will respond to anti-inflammatory medication or steroid infiltration. The latter invariably require operative exploration [8].

Costochondritis is often confused with Tietze syndrome, a

Fig. 4. Histologic findings of infective costochondritis. (A) The surface of the cartilage eroded by neutrophils and necrotic debris (H\&E, $\times 100)$. (B) There are degenerated necrotic debris and neutrophils and inflammatory infiltrate at the cartilage $(H \& E, \times 40)$. 
similar but rare disorder, which is an inflammatory process causing visible enlargement of the costochondral junction [10]. Diagnosis of Tietze syndrome is based on basic clinical trials that exclude other diseases such as coronary diseases and infection of cartilage. It occurs in a single rib $70 \%$ of the time, predominantly in rib two $[7,8]$. One study reported the cause of chest pain in a variant of Tietze syndrome as initial abnormal traction on the insertion of the pectoral muscles to the sternum [3].

Breast augmentation frequently involves an implant being inserted to alter the shape and size of the breast [1]. Although various complications can occur after breast augmentation, infection is one of the more frequently occurring complications and can have a direct and significant impact on the patient since its management may require implant removal. Implant-associated infections generally cause local inflammatory reactions or fibrosis in the surrounding capsule, but perichondrial or deep sternal infiltration, including costochondritis, is a rare problem after breast augmentation surgery.

There have been no reports of life-threatening conditions such as sepsis due to infective costochondritis following breast surgery. But, considering the anatomy of the anterior chest wall, infection of the costal cartilage could affect the pleura and lungs, with further progress being potentially fatal. Therefore, accurate diagnosis and early treatment is important. The differences between infective costochondritis, inflammatory costochondritis and variant Tietze syndrome are relatively clear. However, since costal infections are rare after breast surgery and therefore unfamiliar to many private practitioners, nonspecific physical examination findings and subjective symptoms could readily cause confusion and lead to misdiagnosing infection of peri-implant pectoralis muscle or perichondrium as inflammatory costochondritis or variant Tietze syndrome.

There may be some controversy concerning whether the patient's costochondritis had started as a mild infection misdiagnosed as simple inflammation occurring after breast augmentation, or had newly occurred after implant removal. The patient initially presented no obvious symptoms of infection except pain, and without any active measures including surgical exploration taken to find the cause of pain, the implants were simply removed. Thereafter, the patient was hospitalized and re-operated on only after infection was evident. Based on our experience, we strongly recommend radiologic studies including MRI and computed tomography and early adequate irriga- tion and surgical debridement whenever infective costochondritis is suspected after breast surgery. Future research and discussion will be needed to determine whether there are other effective methods to treat in addition to early surgical procedures in atypical infective costochondritis.

\section{Conflict of interest}

No potential conflicts of interest relevant to this article are reported.

\section{Acknowledgments}

Seok Won Hong https://orcid.org/0000-0002-5634-7017

Da Woon Lee https://orcid.org/0000-0002-6969-5643

Hwan Jun Choi https://orcid.org/0000-0002-0752-0389

\section{References}

1. Johnson M. Breast implants: history, safety, and imaging. Radiol Technol 2013;84:439M-515M.

2. Headon H, Kasem A, Mokbel K. Capsular contracture after breast augmentation: an update for clinical practice. Arch Plast Surg 2015;42:532-43.

3. van Schalkwyk AJ, van Wingerden JJ. A variant of Tietze's syndrome occurring after reconstructive breast surgery. Aesthetic Plast Surg 1998;22:430-2.

4. Carabasi RJ, Christian JJ, Brindley HH. Costosternal chondrodynia: a variant of Tietze's syndrome? Dis Chest 1962;41: 559-62.

5. Moses MA, Banwell PE, Murphy JV, et al. Infective costochondritis following breast reconstruction. Plast Reconstr Surg 2004;114:1356-7.

6. Ayloo A, Cvengros T, Marella S. Evaluation and treatment of musculoskeletal chest pain. Prim Care 2013;40:863-87.

7. Disla E, Rhim HR, Reddy A, et al. Costochondritis: a prospective analysis in an emergency department setting. Arch Intern Med 1994;154:2466-9.

8. Proulx AM, Zryd TW. Costochondritis: diagnosis and treatment. Am Fam Physician 2009;80:617-20.

9. Stedman's Online Medical Dictionary. Costochondritis [Internet]. Stedman's Online; c2009 [cited 2019 Mar 6]. Available from: http://www.stedmansonline.com/index.

10. Fam AG. Approach to musculoskeletal chest wall pain. Prim Care 1988;15:767-82. 